\title{
Propriété immobilière et trajectoires salariales en France
}

Homeownership and Wage Trajectories in France

Carole Brunet, Nathalie Havet et Jean-Yves Lesueur

\section{(2) OpenEdition}

Édition électronique

URL : http://journals.openedition.org/travailemploi/4767

DOI : 10.4000/travailemploi.4767

ISSN : 1775-416X

Éditeur

DARES - Ministère du Travail

\section{Édition imprimée}

Date de publication : 15 décembre 2010

Pagination : 17-27

ISSN : 0224-4365

\section{Référence électronique}

Carole Brunet, Nathalie Havet et Jean-Yves Lesueur, « Propriété immobilière et trajectoires salariales en France », Travail et Emploi [En ligne], 124 | octobre-décembre 2010, mis en ligne le 15 décembre 2012, consulté le 20 avril 2019. URL : http://journals.openedition.org/travailemploi/4767 ; DOI : 10.4000/travailemploi.4767 


\title{
Propriété immobilière et trajectoires salariales en France
}

\author{
Carole Brunet (*), Nathalie Havet (**), Jean-Yves Lesueur (***)
}

L'étude empirique proposée dans cet article s'inscrit dans la lignée des travaux portant sur les effets des choix résidentiels sur la qualité de l'insertion professionnelle sur le marché du travail. Elle se concentre sur la population en emploi et cherche à déterminer l'influence, a priori ambigüe, $d u$ statut résidentiel sur les salaires individuels. D’une part, la propriété immobilière peut, en réduisant la mobilité et la taille du bassin de nouveaux emplois envisageables, diminuer les opportunités d'accroître son salaire ; d'autre part, les propriétaires peuvent faire jouer des réseaux sociaux plus importants et faire valoir une plus grande stabilité professionnelle auprès de leurs employeurs, ce qui est bénéfique pour une promotion salariale interne éventuellement assortie $d u$ financement d'une formation interne. Les résultats obtenus à partir de la partie française du Panel européen des ménages 1995-2001 montrent que les propriétaires bénéficient, toutes choses égales par ailleurs, d'une prime salariale en France par rapport aux locataires. Ces résultats remettent en cause l'hypothèse d'Oswald selon laquelle la propriété immobilière serait néfaste aux performances sur le marché du travail.

Dans la plupart des pays de l'OCDE, les politiques publiques ont depuis la fin de la Deuxième Guerre mondiale généralement poussé au développement de la propriété immobilière. Par exemple, de multiples dispositifs favorisant la possession de sa résidence principale ont été impulsés par les différents gouvernements européens et américains : défiscalisation des intérêts des emprunts immobiliers, baisse des droits de donation - succession, prêts à taux zéro ou encore prêts à taux bonifié en faveur des ménages à faibles revenus (BELOT, EDERVEEN, 2005). Désormais, il est donc plus courant d'être propriétaire de son logement que d'en être locataire dans la zone OCDE (CATTE et al., 2004). La France ne déroge pas à cette tendance : l'accession à la propriété s'est régulièrement accrue, passant de près de $43 \%$ en 1965 à $55 \%$ en 1985 . Elle s'est stabilisée à la fin des années 1980 et durant la première moitié des années 1990, avant de connaître une reprise pour atteindre aujourd'hui $57 \%$ (DUBUJET, Blanc, 2000 ; Daubresse, 2003 ; Bosvieux, 2005).

Néanmoins, ces stratégies publiques qui favorisent la création de «nations de propriétaires » ne modifient pas seulement le marché du logement. Il existe

(*) LED, université Paris 8 ; carole.brunet@ univ-paris8.fr (**) GATE Lyon-Saint-Étienne, université de Lyon ; havet@ gate.cnrs.fr.fr

(***) GATE Lyon-Saint-Étienne, université de Lyon ; lesueur@ gate.cnrs.fr. Les auteurs remercient Carole Herbin, Alexis Penot et Amandine Roche pour leurs précieuses suggestions et aides techniques ainsi que la DARES pour son soutien financier dans le cadre de l'appel à projet «Mobilité Professionnelle». Les opinions exprimées et les analyses développées dans cet article restent propres aux auteurs. des liens étroits entre les choix résidentiels et le comportement des individus sur le marché du travail. Par exemple, le statut résidentiel est susceptible d'influencer la mobilité professionnelle, en conditionnant les offres d'emploi accessibles physiquement à l'individu et en affectant sa mobilité géographique. En effet, la propriété immobilière est associée à des coûts de transaction très élevés par rapport à la location et constitue en ce sens un obstacle à la mobilité. Plus récemment, la crise des subprimes aux ÉtatsUnis et sa diffusion à l'économie réelle à l'échelle internationale ont rappelé les liens étroits entre le secteur immobilier et les autres compartiments de l'économie. Cette crise a également soulevé de façon cruciale la question de la soutenabilité d'une politique économique de promotion de la propriété immobilière déconnectée des aspects économiques réels, notamment ceux relatifs au marché du travail. Ainsi, afin de savoir si une coordination renforcée entre politiques de logement et de l'emploi serait souhaitable, il est indispensable d'avoir une évaluation des répercussions indirectes de ces stratégies sur les performances du marché du travail.

À ce sujet, la littérature économique s'est essentiellement intéressée à l'impact de la propriété immobilière sur les durées de chômage et les mobilités professionnelles (voir Havet, Рenot, 2010, pour une revue de la littérature). La question de l'influence du statut résidentiel sur les salaires a été jusqu'à ce jour délaissée, alors que d'un point de vue théorique, elle est a priori indéterminée. Elle mérite donc d'être tranchée empiriquement, ce que se propose de faire cet article à partir de la partie 
française du Panel européen des ménages 1995 2001 (PEM).

Peut-on mettre en évidence un lien statistique entre statut résidentiel et salaire ? $\mathrm{Si}$ oui, résistet-il à la prise en compte des caractéristiques des individus, de leur poste et de leur entreprise ? Ce sont autant de questions auxquelles nous tenterons d'apporter des éléments de réponse et qui nous semblent à même d'éclairer les politiques en cours sur le marché du travail et du logement. Après avoir présenté les arguments théoriques supposés fonder l'influence du statut résidentiel sur les salaires, nous préciserons la démarche empirique que nous avons adoptée et analyserons les résultats obtenus pour tenter d'enrichir la compréhension des conséquences des politiques favorisant l'accession à la propriété. Nos résultats montrent principalement qu'en France, les propriétaires bénéficient, toutes choses égales par ailleurs, d'une prime salariale par rapport aux locataires, ce qui remet en cause l'hypothèse d'Oswald ( $c f$. infra) selon laquelle la propriété immobilière serait néfaste aux performances sur le marché du travail.

\section{Problématique et hypothèses théoriques}

\section{Propriété immobilière et performances du marché du travail}

Dans le courant de la littérature traitant des interactions entre les marchés de l'emploi et du logement, c'est surtout sur le chômage que l'impact du statut résidentiel a été étudié. Au niveau macro-économique, Oswald (1996, 1998), Belot, VAN OuRs (2001) et NicKELl et al. (2005) montrent que pour les pays de l'OCDE, le taux de propriétaire dans l'économie est significativement et positivement corrélé avec le taux de chômage. L'explication avancée de ce phénomène, connue sous le nom «d'hypothèse d'Oswald », est que les contraintes de mobilité associées à la propriété immobilière dégradent le processus d'appariement sur le marché de l'emploi, ce qui conduit à un taux de chômage plus élevé. Toutefois, les travaux micro-économiques les plus récents et robustes plaident plutôt pour une réfutation de cette hypothèse. Munch et al. (2006) pour le Danemark, vaN VuUren (2009) pour les Pays-Bas et Brunet et al. (2011) pour la France, trouvent que le fait d'être propriétaire réduit les durées des épisodes de chômage. B ATTU et al. (2008) et BRUNET et al. (2007, 2011), respectivement pour le Royaume-Uni et les États-Unis, concluent quant à eux qu'il n'existerait pas de différence significative entre propriétaires et locataires pour les sorties de chômage.

Ces dernières années, des études portant sur le lien entre statut résidentiel et stabilité dans l'emploi ont cherché à vérifier si la propriété immobilière était bien une entrave aux mobilités professionnelles des individus en emploi. En effet, le statut de propriétaire, associé à des coûts de transaction très élevés par rapport au statut de locataire, devrait constituer un obstacle à la recherche d'un meilleur emploi pour les actifs et donc limiter les changements d'emploi. Les propriétaires devraient ainsi enregistrer des durées d'emploi plus longues. Plus précisément, les propriétaires devraient d'autant moins connaître de transitions professionnelles que, situés en haut de l'échelle des salaires, ils subissent un coût associé à l'abandon de leur emploi plus élevé (Burgess, 1992). Ils sont également susceptibles d'avoir effectué plus de dépenses en logement que les locataires, montant qui influence positivement les coûts de mobilité en cas de relocalisation (VAN DEN BERG, 1990). DE GrAaf et al. (2007, 2009), MuNCH et al. (2008) et BATTU et al. (2008) confirment empiriquement pour un certain nombre de pays que les propriétaires ont des durées d'emploi plus longues que leurs homologues locataires : les propriétaires ont moins de chances de se retrouver au chômage (environ $25 \%$ de moins) que les locataires dans la même situation professionnelle et ont des taux de changement d'emploi inférieurs de l'ordre de 20 à $30 \%$.

Les études récentes mettent donc en évidence une position relativement plus favorable des propriétaires sur le marché du travail, que ce soit face au risque de chômage ou en termes de stabilité dans l'emploi, position bénéfique aux progressions de carrières internes. Toutefois, il reste à vérifier si ces conclusions en faveur de la propriété immobilière ne seraient pas à relativiser lorsque l'on considère la qualité des appariements des propriétaires sur le marché de l'emploi. Pour les chômeurs, le retour à l'emploi plus rapide des propriétaires pourrait simplement refléter qu'ils sont prêts à faire davantage de sacrifices en termes de salaires et de postes occupés. Par exemple, on peut supposer que les propriétaires encore en charge d'emprunt immobilier à rembourser aient tendance à manifester, lors de leur prospection, des salaires de réserve plus bas, à la fois pour accélérer leur sortie du chômage en période d'endettement et pour retrouver un emploi à proximité d'un domicile récemment acquis, dont ils souhaitent conserver les aménités. Pour la population salariée, la plus grande stabilité dans l'emploi des propriétaires pourrait limiter le bon déroulement de leurs carrières. Notamment, en refusant tout nouvel emploi impliquant une mobilité résidentielle, ils pourraient se priver d'un ensemble d'opportunités salariales intéressantes. C'est pourquoi, l'étude de l'influence de la propriété immobilière sur les salaires, qui représentent un indicateur de la qualité de l'appariement de l'individu avec son emploi, est primordiale. 


\section{Quels effets attendus du statut résidentiel sur les salaires?}

Les arguments théoriques proposés par Coulson et Fisher (2002, 2009) et Munch et al. (2008) reposent sur des hypothèses particulières quant à l'influence de la propriété immobilière sur le chômage et la stabilité dans l'emploi, et impliquent des effets contradictoires sur les salaires.

COUlson et Fisher (2002) considèrent comme hypothèse de départ que les propriétaires ont des durées de chômage plus élevées pour prédire que les salaires des propriétaires devraient être plus faibles que ceux des locataires. En effet, selon la théorie de la négociation salariale, si la probabilité et la durée de chômage sont plus élevées pour les propriétaires, les employeurs devraient en tirer avantage dans la négociation (diminution du pouvoir de négociation des propriétaires) et ainsi leur offrir des salaires plus faibles. Coulson et FISHER (2009) prédisent un impact négatif de la propriété immobilière sur les salaires à partir de deux justifications plus réalistes. D'une part, ils montrent, à l'aide d'un modèle de recherche d'emploi, que les salaires des locataires devraient être plus élevés, puisque leur bassin de recherche d'emplois est plus vaste ; les propriétaires sont au contraire plus réfractaires à se tourner vers le marché de l'emploi non-local qui nécessiterait un changement de résidence. D'autre part, ils avancent l'hypothèse que les propriétaires et les locataires ne retirent pas la même utilité du chômage. En particulier, les propriétaires sont supposés être désavantagés par rapport aux locataires : une fois au chômage, il leur est plus difficile et coûteux d'ajuster leur consommation liée au logement avec leurs ressources. Conditionnellement à être en emploi, les propriétaires accepteraient en conséquence, toutes choses égales par ailleurs, des salaires plus faibles que les locataires, du fait d'un coût d'opportunité de l'état de chômage plus élevé.

En revanche, Munch et al. (2008) partent de l'hypothèse que les propriétaires changent moins souvent d'emploi que les locataires. Ils supposent implicitement que les employeurs connaissent le statut résidentiel de leurs salariés et qu'ils en tiennent compte dans leur processus de détermination des salaires. La plus forte stabilité dans l'emploi des propriétaires, appréciée par les entreprises, leur conférerait un plus grand pouvoir de négociation salariale. En particulier, du point de vue de l'entreprise, l'espérance de la valeur actualisée d'un emploi serait plus élevée quand celui-ci est occupé par un propriétaire plutôt que par un locataire, les périodes de vacances de l'emploi étant plus réduites. Les employeurs préféreraient ainsi embaucher des propriétaires, toutes choses égales par ailleurs. Ils pourraient même leur proposer des salaires plus élevés afin de les attirer. En outre, cet écart de salaire risque de perdurer au fur et à mesure du déroulement des carrières. En effet, les propriétaires devraient bénéficier d'un niveau de capital humain spécifique plus élevé puisqu'ils ont une durée supposée plus longue pour rentabiliser leur investissement du fait de leur plus forte stabilité dans l'emploi. Ceci se traduirait par une productivité moyenne plus importante pour les propriétaires, justifiant des primes salariales persistantes en faveur des propriétaires.

Toutefois, à partir de la même hypothèse de départ de Munch et al. (2008), nous pourrions conclure à des effets opposés. À leurs arguments, nous pourrions effectivement ajouter que les propriétaires en emploi sont moins enclins, en raison de coûts de mobilité élevés, à en chercher un nouveau : ils se priveraient par là même d'offres d'emploi intéressantes améliorant la qualité de leur appariement et donc indirectement leur salaire. Cette interprétation peut également être renforcée par les travaux développés sur la formation non concurrentielle des salaires (MANning, 2003). En effet sur un marché local, si l'augmentation du nombre de propriétaires réduit la mobilité des travailleurs, les entreprises pourraient tirer avantage de ces coûts de friction sur le bassin d'emploi pour pratiquer des salaires en dessous de la référence concurrentielle. Ainsi pourrait apparaître une discrimination salariale à l'égard des propriétaires.

Néanmoins, on ne peut pas non plus ignorer que la plus faible mobilité des propriétaires les amènent à s'investir davantage dans leur milieu social et leur permet de bénéficier de réseaux sociaux plus importants, propices à de bonnes opportunités professionnelles. En conséquence, l'influence de la propriété immobilière sur les salaires, que ce soit via les mécanismes de négociation salariale ou via les comportements de mobilités réduites qui lui sont associés, est ambiguë. C'est pourquoi, nous aurons recours à une évaluation économétrique pour essayer de savoir si ces effets théoriques opposés se compensent et dans le cas contraire, lequel des deux domine.

\section{Sources et méthodes}

\section{Sources statistiques}

Nous nous proposons, pour examiner l'impact de la propriété immobilière sur les salaires, d'utiliser les données des sept dernières vagues 1995-2001 de la partie française du PEM (1). Cette enquête longitudinale annuelle auprès d'un échantillon représentatif a la particularité d'être un panel d'individus : chacune des personnes du ménage a été interrogée une première fois, puis a été suivie d'une année à l'autre même en cas de déménagement ou de changement dans la composition du ménage. Dans les panels de logement, comme l'enquête Emploi, les logements

(1) Voir BreuIL-GRENIER, VALDELIEVRe (2001) pour plus de détails. 
sont réenquêtés, que leurs occupants aient changé de domicile ou non : une part importante de l'échantillon correspond donc à de nouveaux enquêtés et seules les trajectoires d'activité des personnes n'ayant jamais déménagé peuvent être reconstituées. Un des avantages du PEM pour notre étude est ainsi de limiter le biais potentiel lié à l'utilisation d'un sous-échantillon de personnes n'ayant jamais déménagé et d'avoir une meilleure représentativité des différents statuts résidentiels par rapport à l'ensemble de la population. Par ailleurs, cette enquête recueille auprès des individus des données sur leurs salaires, leurs heures de travail, leur capital humain, les caractéristiques de leur emploi et autres informations démographiques habituellement disponibles dans les enquêtes auprès des ménages (âge, situation matrimoniale, niveau de scolarité, milieu social, etc.). Il en résulte une base de données riche de renseignements interreliés sur les comportements vis-à-vis du marché du travail et du marché du logement. Toutefois, le PEM ne contient pas de renseignements précis sur le lieu de résidence hormis le département et la taille de l'unité urbaine.
Notre étude empirique sur les salaires est bien évidemment basée sur la seule population en emploi, avec une activité salariée. Plus précisément, seuls les individus du PEM âgés d'au moins 17 ans et de moins de 60 ans, et vivant dans un ménage de type conventionnel ont été retenus. Nous excluons les ménages constitués de familles impliquant des ascendants indirects ou plus d'un ascendant direct, ainsi que les enfants vivant encore au domicile parental, et dont les trajectoires tant sur le marché de l'emploi que du logement relèvent d'une logique d'insertion spécifique (LAFÉRRÈre, 2005). Enfin, nous avons exclu les locataires des logements sociaux du secteur public (HLM, etc.) dont les parcours sur le marché du travail sont atypiques et méritent d'être analysés séparément. Il nous reste alors 11489 observations parfaitement renseignées, correspondant à 2909 individus actifs du PEM. Quelques statistiques descriptives de notre sous-échantillon sont reportées dans le tableau 1.

\section{Tableau 1 : Statistiques descriptives}

\begin{tabular}{|c|c|c|}
\hline & Locataires & Propriétaires \\
\hline Salaire horaire moyen (euros) & 8,33 & 10,26 \\
\hline Individus ayant reçu un héritage & $3,4 \%$ & $5,7 \%$ \\
\hline \multicolumn{3}{|l|}{ Âge } \\
\hline $17-25$ ans & $4,0 \%$ & $0,2 \%$ \\
\hline $26-35$ ans & $31,6 \%$ & $12,1 \%$ \\
\hline $36-45$ ans & $34,0 \%$ & $40,6 \%$ \\
\hline Plus de 45 ans & $30,4 \%$ & $47,1 \%$ \\
\hline \multicolumn{3}{|l|}{ Situation familiale } \\
\hline Couples sans enfant & $20,1 \%$ & $18,9 \%$ \\
\hline Couples avec enfant(s) & $65,4 \%$ & $75,6 \%$ \\
\hline Familles monoparentales & $12,5 \%$ & $3,1 \%$ \\
\hline Personnes seules & $2,0 \%$ & $2,4 \%$ \\
\hline \multicolumn{3}{|l|}{ Niveau de diplôme } \\
\hline Aucun diplôme & $38,6 \%$ & $32,8 \%$ \\
\hline Diplôme d'enseignement technique court & $37,0 \%$ & $35,4 \%$ \\
\hline Diplôme d'enseignement technique long & $12,1 \%$ & $14,9 \%$ \\
\hline Diplôme d'enseignement supérieur premier cycle & $7,5 \%$ & $10,0 \%$ \\
\hline Diplôme d'enseignement supérieur deuxième cycle & $4,8 \%$ & $6,9 \%$ \\
\hline \multicolumn{3}{|l|}{ Expérience professionnelle } \\
\hline Moins de 5 ans & $3,9 \%$ & $0,4 \%$ \\
\hline $6-10$ ans & $13,5 \%$ & $2,8 \%$ \\
\hline $11-20$ ans & $30,8 \%$ & $21,7 \%$ \\
\hline Plus de 20 ans & $51,8 \%$ & $75,1 \%$ \\
\hline \multicolumn{3}{|l|}{ Catégorie socioprofessionnelle } \\
\hline Professions intellectuelles et intermédiaires & $26,1 \%$ & $37,4 \%$ \\
\hline Employés & $37,4 \%$ & $34,6 \%$ \\
\hline Ouvriers & $36,5 \%$ & $28,0 \%$ \\
\hline Contrat à durée indéterminée & $89,5 \%$ & $94,9 \%$ \\
\hline Nombre d'observations & 3174 & 8315 \\
\hline
\end{tabular}




\section{Analyse descriptive}

Ce tableau met en évidence qu'il existe une différence de salaire horaire en faveur des propriétaires. L'écart de salaire horaire moyen entre propriétaires et locataires s'élèverait à 23,1 \% en France. Il semblerait donc que l'accession à la propriété soit associée à une prime salariale. Toutefois, propriétaires et locataires ne constituent pas deux populations homogènes, et se distinguent au contraire par certaines caractéristiques individuelles. En effet, les ménages souhaitant acquérir un bien immobilier se voient généralement imposer par les banques et les autres organismes de crédit deux types de conditions : l'une porte sur le montant de l'apport initial puisque les ménages ne peuvent emprunter plus d'une certaine proportion de la valeur du bien immobilier convoité ; l'autre porte sur le niveau de revenu du ménage par rapport à la charge de remboursement que représente l'emprunt contracté. Compte tenu de ces restrictions institutionnelles, il n'est pas surprenant de constater que les propriétaires sont, en moyenne, plus âgés et légèrement plus diplômés ; qu'ils occupent davantage des emplois à durée indéterminée et ont une expérience sur le marché du travail plus longue que les locataires. Par conséquent, l'écart salarial observé entre propriétaires et locataires n'est pas entièrement attribuable au seul effet propre du statut résidentiel : il ne tient pas compte des effets conjoints des autres variables individuelles telles que l'âge ou l'expérience sur le marché du travail sur l'accès à la propriété et le niveau des salaires. Il est donc nécessaire de recourir à un modèle économétrique pour obtenir une comparaison contrôlée de l'impact du statut résidentiel sur les salaires, toutes choses égales par ailleurs.

\section{Méthodes économétriques}

Nous basons notre estimation économétrique sur une équation de salaire standard à la MINCER (1974). Nous traitons le logarithme du salaire horaire comme variable expliquée et le fait d'être propriétaire de son logement comme une variable explicative. Toutefois, il est très probable que cette variable de propriété immobilière soit endogène car il existe aussi certainement un impact causal des salaires sur le statut résidentiel. En effet, la propriété immobilière, notamment pour ceux qui sont obligés de faire un emprunt pour y accéder, est conditionnée par le niveau des ressources financières et des flux de revenus, et donc par les salaires. Afin d'éviter que l'évaluation n'en soit biaisée, nous recourons à une technique d'estimation par variables instrumentales, au lieu des traditionnels moindres carrés ordinaires. Plus précisément, nous n'introduisons pas directement une variable dichotomique représentant le statut résidentiel dans l'équation de salaire : nous la remplaçons par la probabilité individuelle d'être propriétaire, estimée à l'aide d'un probit dans une étape antérieure, afin de pallier son éventuel caractère endogène $(2)$ ( $c f$. annexe).

Cette méthode des variables instrumentales impose certaines contraintes d'identification. Les paramètres de l'équation de salaire ne peuvent pas être identifiés si les déterminants des salaires incluent tous les déterminants du statut résidentiel. La contrainte d'identification est qu'au moins une variable explicative des décisions de propriété immobilière - appelée instrument - soit exclue de l'équation de salaire. Ici, cette contrainte est vérifiée : les « instruments » retenus par notre étude sont une variable indiquant si l'individu a reçu un héritage au cours de l'année précédente et des variables reflétant les montants moyens des taxes locales (taxe foncière et taxe d'habitation), calculées par taille d'unité urbaine et département. La variable d'héritage influence le choix de statut résidentiel mais pas directement le niveau des salaires perçus. Le montant des héritages étant loin d'être toujours très élevé, les individus qui en ont bénéficié ne modifient généralement pas leur comportement sur le marché du travail et peuvent utiliser cet apport financier pour concrétiser un projet immobilier. On espère à travers les variables de taxes locales représenter les aménités liées au lieu de résidence, qui sont censées augmenter la probabilité d'être propriétaire de son logement sans pour autant modifier les niveaux de salaire individuels. Mais ces variables de taxes locales peuvent également capter un effet de coût de logement, qui jouerait négativement sur l'accession à la propriété. Sur nos données, leur impact global est significatif et positif, ce qui traduit l'importance de la qualité des services publics et des infrastructures dans le choix du statut résidentiel.

Concernant les déterminants additionnels des salaires, nous retenons des variables reflétant le capital humain du salarié (ancienneté, expérience, diplôme), sa situation familiale, le type d'emploi occupé (profession, secteur d'activité, nature du contrat). Nous incluons également le sexe et la nationalité afin de tenir compte des discriminations potentielles à l'encontre des femmes et des étrangers lors de la détermination des salaires. Comme les salaires peuvent être influencés par le coût de la vie, nous avons introduit dans les régressions des variables caractérisant la taille de l'unité urbaine du lieu de résidence de l'individu.

\section{Résultats empiriques}

Le tableau 2 reporte les résultats des estimations des équations de salaires effectuées sur données françaises, respectivement en contrôlant ou non le caractère endogène de la propriété immobilière.

(2) Pour une présentation détaillée de la méthode des variables instrumentales, voir WoOLDRIDGE (2002, chapitre 5). 
Tableau 2 : Déterminants des salaires, estimations par la méthode des moindres carrés ordinaires (MCO) et des variables instrumentales (VI)

\begin{tabular}{|c|c|c|c|}
\hline Variables & Catégories & $\begin{array}{l}\text { Pas d'endogénétié } \\
\text { supposée (MCO) }\end{array}$ & \begin{tabular}{|c|} 
Avec endogénéité supposée \\
(VI)
\end{tabular} \\
\hline \multicolumn{2}{|l|}{ Constante } & $3,343^{(* * * *)}$ & 3,349 (****) \\
\hline Statut résidentiel & $\begin{array}{l}\text { Locataire privé } \\
\text { Propriétaire }\end{array}$ & $\begin{array}{l}\text { Réf. } \\
0,055^{(* * * *)}\end{array}$ & $\begin{array}{l}\text { Réf. } \\
0,145^{(* * * *)}\end{array}$ \\
\hline Sexe & $\begin{array}{l}\text { Homme } \\
\text { Femme }\end{array}$ & $\begin{array}{c}\text { Réf. } \\
-0,153^{(* * * *)}\end{array}$ & $\begin{array}{c}\text { Réf. } \\
-0,158^{(* * * *)}\end{array}$ \\
\hline Nationalité & $\begin{array}{l}\text { Étrangère } \\
\text { Française }\end{array}$ & $\begin{array}{l}\text { Réf. } \\
0,078^{(* * * *)}\end{array}$ & $\begin{array}{l}\text { Réf. } \\
0,069^{(* * * *)}\end{array}$ \\
\hline $\begin{array}{l}\text { Structure } \\
\text { familiale }\end{array}$ & $\begin{array}{l}\text { Couple sans enfants } \\
\text { Couple avec enfant(s) } \\
\text { Famille monoparentale } \\
\text { Autre type de famille } \\
\text { Autre type de ménage } \\
\end{array}$ & $\begin{aligned} & \text { Réf. } \\
&-0,015 \\
& 0,011 \\
&-0,050^{(* *)} \\
&-0,165^{(* * * *)} \\
&\end{aligned}$ & $\begin{array}{c}\text { Réf. } \\
-0,020^{(* *)} \\
0,037^{(*)} \\
-0,060^{(* *)} \\
-0,165^{(* * * *)} \\
\end{array}$ \\
\hline $\begin{array}{l}\text { Statut du } \\
\text { conjoint }\end{array}$ & $\begin{array}{l}\text { Pas de conjoint en activité } \\
\text { En emploi } \\
\text { Au chômage }\end{array}$ & $\begin{array}{l}\text { Réf. } \\
-0,039 * * * * \\
-0,028(*)\end{array}$ & $\begin{array}{l}\text { Réf. } \\
-0,045^{(* * * *)} \\
-0,024^{(*)}\end{array}$ \\
\hline Diplôme & $\begin{array}{l}\text { Aucun ou primaire } \\
\text { Enseignement technique court } \\
\text { Enseignement technique long, bac général } \\
\text { Enseignement supérieur court } \\
\text { Enseignement supérieur long }\end{array}$ & $\begin{array}{l}\text { Réf. } \\
0,030^{(* * * *)} \\
0,211^{(* * * *)} \\
0,235^{(* * * *)} \\
0,334(* * * *) \\
\end{array}$ & $\begin{array}{l}\text { Réf. } \\
0,025^{(* * * *)} \\
0,199^{(* * * *)} \\
0,223^{(* * * *)} \\
0,322^{(* * * *)}\end{array}$ \\
\hline Profession & $\begin{array}{l}\text { Employé } \\
\text { Ouvrier } \\
\text { Profession intellectuelle } \\
\text { Profession intermédiaire }\end{array}$ & $\begin{array}{l}\text { Réf. } \\
-0,006 \\
0,464 \text { (****) } \\
0,243^{(* * * *)}\end{array}$ & $\begin{array}{l}\text { Réf. } \\
-0,005 \\
0,456^{(* * * *)} \\
0,239 \text { (****) }\end{array}$ \\
\hline \multicolumn{2}{|l|}{ Expérience } & $0,011^{(* * * *)}$ & 0,007 (****) \\
\hline \multicolumn{2}{|l|}{ Expérience au carré } & $-3,10^{-4(* * * *)}$ & $-2,10^{-4(* * * *)}$ \\
\hline \multicolumn{2}{|l|}{ Ancienneté } & $0,016^{(* * * *)}$ & $0,016^{(* * * *)}$ \\
\hline \multicolumn{2}{|l|}{ Ancienneté au carré } & $-0,001^{(* * *)}$ & $-1,10^{-4(* *)}$ \\
\hline Temps de travail & $\begin{array}{l}\text { Temps partiel } \\
\text { Temps complet }\end{array}$ & $\begin{array}{c}0,016 \\
\text { Réf. }\end{array}$ & $\begin{array}{l}0,020^{(*)} \\
\text { Réf. }\end{array}$ \\
\hline CDI & $\begin{array}{l}\text { Oui } \\
\text { Non }\end{array}$ & $\begin{array}{l}0,069^{(* * * *)} \\
\text { Réf. }\end{array}$ & $\begin{array}{l}0,061^{(* * * *)} \\
\text { Réf. }\end{array}$ \\
\hline Secteur & $\begin{array}{l}\text { Privé } \\
\text { Public }\end{array}$ & $\begin{array}{l}\text { Réf. } \\
0,136^{(* * * *)}\end{array}$ & $\begin{array}{l}\text { Réf. } \\
0,131^{(* * * *)}\end{array}$ \\
\hline Secteur d'activité & $\begin{array}{l}\text { Commerce } \\
\text { Industrie agricole, alimentaire, biens de consommation } \\
\text { Industrie automobile et biens d'équipement } \\
\text { Industrie biens intermédiaires } \\
\text { Énergie, construction } \\
\text { Transport } \\
\text { Finance, immobilier } \\
\text { Services aux entreprises } \\
\text { Éducation, services aux particuliers } \\
\text { Administration }\end{array}$ & $\begin{array}{l}\text { Réf. } \\
0,120^{(* * * *)} \\
0,149^{(* * * *)} \\
0,115^{(* * * *)} \\
0,114^{(* * * *)} \\
0,084^{(* * * *)} \\
0,073^{(* * * *)} \\
0,078^{(* * * *)} \\
0,001 \\
-0,034(*)\end{array}$ & $\begin{array}{l}\text { Réf. } \\
0,124(* * * *) \\
0,148^{(* * * *)} \\
0,113^{(* * * *)} \\
0,114(* * * *) \\
0,089^{(* * * *)} \\
0,079^{(* * * *)} \\
0,077^{(* * * *)} \\
0,004 \\
-0,030^{(*)}\end{array}$ \\
\hline \multicolumn{2}{|l|}{ Chômage local } & $-0,001$ & $-0,001$ \\
\hline Taille de l'unité urbaine & $\begin{array}{l}<5000 \text { habitants } \\
5000-10000 \text { habitants } \\
10000-20000 \text { habitants } \\
20000-50000 \text { habitants } \\
50000-100000 \text { habitants } \\
100000-200000 \text { habitants } \\
200000-2000000 \text { habitants } \\
\text { Agglomération parisienne }\end{array}$ & $\begin{array}{l}0,029^{(* *)} \\
-0,003 \\
0,009 \\
0,017 \\
0,026^{(*)} \\
-0,007 \\
0,025^{(* *)} \\
0,170^{(* * *)} \\
\end{array}$ & $\begin{array}{l}0,033^{(* *)} \\
0,009 \\
0,024 \\
0,040^{(* *)} \\
0,043^{(* *)} \\
0,009 \\
0,045^{(* * *)} \\
0,196^{(* * *)} \\
\end{array}$ \\
\hline \multicolumn{2}{|l|}{ Nombre d'observations } & 11489 & 11489 \\
\hline
\end{tabular}

Note : + contrôle des différences entre les années.

${ }^{(*)}$ Significatif au seuil de $10 \%,{ }^{(* *)}$ significatif au seuil de $5 \%,{ }^{(* * *)}$ significatif au seuil de $1 \%$, ${ }^{(* * *)}$ significatif au seuil de $0,1 \%$.

Source : Panel européen des ménages 1995-2001, calculs des auteurs. 


\section{Les déterminants du salaire}

Nous retrouvons les mêmes déterminants, que l'équation de salaire soit estimée par la méthode des moindres carrés ou par la méthode des variables instrumentales. De nombreuses variables sont statistiquement significatives et ont l'effet attendu sur les salaires. Être une femme, ainsi qu'être de nationalité étrangère influence négativement le niveau de salaire. Ces résultats sont cohérents avec les phénomènes de discrimination « pure » et «statistique » mis en évidence à l'encontre de ces groupes de salariés lors de la détermination des salaires (BLAU, Ferber 1987 ; Oettinger, 1996 ; Meurs, Ponthieux, 2000 ; HaVET, 2004). À caractéristiques équivalentes, un homme reçoit un salaire horaire supérieur d'environ $17 \%$ à celui d'une femme en France (3). De même, les individus de nationalité étrangère perçoivent des salaires inférieurs d'environ $7 \%$.

Conformément aux théories du capital humain (Mincer, 1974) et de l'appariement (Jovanovic, 1979) les salaires sont croissants avec le niveau de scolarité, l'expérience professionnelle et l'ancienneté dans l'entreprise. Par exemple, les diplômés de l'enseignement technique court ont des salaires en moyenne 2,5\% plus élevés que les personnes ayant au mieux un diplôme de l'enseignement primaire, tandis que les diplômés de l'enseignement supérieur de deuxième cycle ont des salaires supérieurs de $38 \%$.

Les caractéristiques de l'emploi occupé par l'individu jouent aussi sur le niveau de salaire. De façon non surprenante, nous pouvons noter une hiérarchie salariale parmi les professions. En France, les professions intermédiaires et les professions intellectuelles correspondent à des salaires plus élevés que ceux des employés, avec des primes respectives de $27 \%$ et $58 \%$. Les ouvriers n'ont quant à eux ni supplément, ni pénalité en termes de salaire par rapport aux employés, toutes choses égales par ailleurs. Il apparaît aussi que les secteurs d'activité se différencient par le niveau des salaires offerts, notamment du fait de conventions collectives propres à chacun d'eux, de la taille moyenne des entreprises les composant, voire de politiques salariales incitatives spécifiques. Il semble qu'une majorité de secteurs (notamment l'industrie, l'énergie, la construction, le transport, la finance, l'immobilier et les services aux entreprises) offre des salaires supérieurs à ceux du commerce (primes salariales allant de $8 \%$ à $16 \%$ ), tandis que les secteurs de l'éducation, des services aux particuliers et de l'administration offrent des salaires identiques. Enfin, nos données mettent en évidence que la nature du contrat de travail a également un

(3) Les régressions effectuées ont comme variable dépendante le logarithme du salaire. Ainsi, l'influence du genre de l'individu sur le niveau de salaire est mesurée par exp $(0,158)-1$ $=0,171$. Les impacts pour les autres variables sont calculés de la même manière. impact sur les rémunérations individuelles : les salariés en contrat à durée indéterminée ont des salaires supérieurs d'environ $6 \%$ aux salariés en contrat à durée déterminée. En revanche, le fait d'être à temps partiel n'a aucun impact sur le salaire horaire.

Enfin, les individus dont le conjoint est en emploi ont des salaires inférieurs (de $5 \%$ environ) aux individus dont le conjoint n'est pas en activité, tandis qu'aucune différence n'émerge si le conjoint est au chômage. Nous pouvons penser que l'apport d'un second salaire dans un ménage n'incite pas un individu à négocier un salaire plus élevé pour pouvoir subvenir aux besoins du ménage. Par ailleurs, parmi les ménages dont l'un des conjoints n'est pas en activité, il est fort probable que cette décision de non-participation au marché du travail ait été prise parce que le salaire de l'autre conjoint était perçu comme suffisamment élevé pour l'ensemble des besoins de la famille. Nos résultats sur les déterminants du salaire sont globalement en adéquation avec les prédictions théoriques et conformes à la littérature empirique sur le sujet.

\section{Évaluation de l'impact du statut résidentiel sur les salaires}

Il nous reste à répondre à notre question principale sur les liens entre statut résidentiel et salaire. Selon la méthode d'estimation retenue (MCO ou variables instrumentales), le coefficient associé à la propriété immobilière varie énormément. Il nous faut donc trancher entre les deux estimations, ce qui revient à tester si la variable de propriété immobilière est exogène ou endogène dans l'équation de salaire. L'hypothèse testée est : conditionnellement au fait que nous acceptions la validité des instruments (4), pouvons-nous accepter que la variable de statut résidentiel supposée a priori endogène soit en fait exogène ? Ce test est effectué par le biais de la régression augmentée basée sur le test de spécification de HaUSMAN (1978) (cf. annexe). Son application à nos données nous conduit à rejeter l'hypothèse nulle d'exogénéité. Ceci signifie que si nous croyons à la validité de nos instruments, nous ne pouvons pas croire à l'exogénéité de la variable de statut résidentiel. En conséquence, l'impact de la propriété immobilière sur les salaires doit être mesuré à l'aide de la méthode par variables instrumentales et non par la méthode des moindres carrés ordinaires, qui donne des estimations biaisées.

Pour savoir si les propriétaires perçoivent une prime salariale par rapport à leurs homologues locataires, nous devons regarder les coefficients associés à la variable de propriété immobilière. Avec les données du PEM, la variable de statut résidentiel

(4) Dans notre cas, la validité a été vérifiée en testant leur significativité dans la régression probit du statut résidentiel et par le test de suridentification de Sargan. 
est statistiquement significative, suggérant que la propriété immobilière a bien un impact direct sur les salaires en France. Son coefficient étant positif, les propriétaires bénéficieraient, toutes choses étant égales par ailleurs, de salaires en moyenne plus élevés que les locataires. Cette conclusion va dans le même sens que ce que pouvaient laisser entendre les statistiques descriptives du tableau 2. Cependant, la prime salariale moyenne accordée aux propriétaires à caractéristiques équivalentes s'élèverait à 15,6\% et non à $23 \%$ comme pouvaient le suggérer les statistiques descriptives. Ce résultat économétrique nous permet de vérifier qu'une partie de l'écart entre le salaire moyen des propriétaires et celui des locataires était imputable aux différences de caractéristiques (âge, expérience, etc.) entre les deux groupes ; mais qu'une fois ces différences contrôlées, un écart de salaire conséquent persiste entre propriétaires et locataires. En ignorant le problème d'endogénéité du statut résidentiel et donc en se basant sur l'estimation par moindres carrés ordinaires, nous aurions largement sous-estimé l'effet de la propriété immobilière sur les salaires (une prime de 5,5\% au lieu de 15,6\% en faveur des propriétaires). Cela confirme le biais qu'entraînerait la non-prise en compte de l'endogénéité. Toutefois, le sens du biais peut paraître un peu surprenant. A priori, on aurait pu s'attendre à ce que la correction de l'endogénéité diminue l'impact estimé de la propriété immobilière sur les salaires puisque les propriétaires ont en général dû faire preuve de revenus réguliers suffisants pour accéder au crédit immobilier des institutions financières. Nos résultats montrent que les effets de sélection qui se manifestent à travers le coefficient de l'estimation par MCO n'agissent pas nécessairement à la hausse sur le niveau de salaire. Une explication possible est que sur la période étudiée, on a assisté en France à la mise en place et au succès de prêt à taux zéro, qui a entre autres favorisé les ménages modestes dans l'accession à la propriété (GoBILLON, LE BLANC, 2005). Une partie de la sélection des propriétaires s'est donc effectuée sur d'autres critères que des revenus élevés, remettant en cause le sens du biais initialement supposé.

L'impact positif du statut résidentiel sur les salaires, obtenu pour la France, avait déjà été mis en évidence par Munch et al. (2008) pour le Danemark. À partir d'un système d'équations simultanées modélisant les durées d'emploi, les salaires et les choix d'occupation de logement, ces derniers évaluaient la prime salariale en faveur des propriétaires danois de l'ordre de $5 \%$. Il n'est pas surprenant que les estimations économétriques montrent que la propriété immobilière a un effet plus prononcé en France qu'au Danemark, puisque l'écart de salaire moyen entre propriétaires et locataires était initialement moins marqué au Danemark (15\% contre $23 \%$ pour la France). Une partie de la différence observée entre la France et le Danemark peut aussi être imputée aux spécifications économétriques retenues : la modélisation utilisée par MuNCH et al. (2008) est susceptible de mieux capter l'hétérogénéité inobservée. Par ailleurs, notre étude comme celle de MuNCH et al. (2008), en se restreignant à la seule population en emploi, est sujette à un biais potentiel de sélection. Ce dernier existe si les caractéristiques des individus en emploi sont significativement différentes du reste de la population (chômeurs et inactifs). Or, les études indiquent habituellement que la population en emploi est par exemple plus éduquée et a une expérience sur le marché du travail plus importante que celle sans emploi. De même, du fait de ces observations et des conditions d'accès au crédit immobilier en France, les propriétaires ont une plus forte propension à être en emploi que les locataires. En raison de ce processus de sélection dans l'emploi et de caractéristiques individuelles inobservées telles que la motivation de l'individu, ses qualités d'orateur ou de négociateur, qui pourraient jouer positivement à la fois sur l'obtention d'un emploi, le niveau de salaires et l'accession à la propriété, la prime salariale en faveur des propriétaires pourrait être légèrement surévaluée dans notre traitement économétrique sur données françaises.

L'association positive observée entre propriété immobilière et salaire va dans le sens des arguments théoriques proposés par MuNCH et al. (2008) et à l'encontre de l'hypothèse d'Oswald. Certes les propriétaires français, par leur moindre mobilité, se priveraient d'un ensemble d'offres d'emplois et de salaires potentiellement intéressantes. Mais cet effet négatif sur les rémunérations serait plus que compensé par leur forte stabilité dans l'emploi, récompensée financièrement par les employeurs. En particulier, nous pouvons imaginer que les propriétaires français accumulent plus de capital humain spécifique et ont davantage accès à la formation continue en entreprise, source en général de hausses de salaire. Par ailleurs, les avantages liés à leurs réseaux sociaux mieux implantés peuvent aussi contribuer à ce que l'effet négatif de leur plus faible mobilité soit compensé. Enfin l'hypothèse de discrimination salariale de la part des employeurs vis-à-vis de salariés propriétaires moins mobiles peut également être réfutée sur la base de nos résultats. En conséquence, le statut de propriétaire et la stabilité dans l'entreprise qui lui est associée seraient des facteurs favorables aux travailleurs du point de vue de la négociation salariale en France.

\section{$*$ \\ * * *}

Cet article s'inscrit dans la lignée des travaux sur les liens entre marché du travail et marché du logement. Il porte plus particulièrement sur les effets salariaux de la propriété immobilière, aspect encore relativement peu étudié au sein de cette littérature 
et jamais à notre connaissance sur données françaises. Nous mobilisons un échantillon issu de la partie française du PEM (1995-2001), afin d'étudier les liens entre statut de propriétaire et niveau de salaire horaire au niveau micro-économique. Plus spécifiquement, nous cherchons à évaluer si la propriété immobilière constitue un obstacle à la carrière salariale, compte tenu des obstacles à la mobilité qu'elle induit, ou si au contraire il existe un effet positif de ce statut résidentiel sur les salaires du fait de la stabilité associée et de l'accumulation de capital humain spécifique qu'elle permet. Alors que les statistiques descriptives mettent en évidence l'existence d'une prime salariale de $23 \%$ pour les individus propriétaires en France, l'enjeu de l'estimation économétrique est d'obtenir une évaluation toutes choses égales par ailleurs, tout en tenant compte du caractère endogène du choix du statut résidentiel.

Une équation de salaire dans laquelle la variable de propriété immobilière est instrumentée a ainsi été estimée. Ses principaux enseignements nuancent mais confirment les observations précédentes, puisque les propriétaires français sont caractérisés par des niveaux de salaire supérieurs de $15 \%$ à ceux des locataires.

Ainsi, en France, les propriétaires semblent bénéficier de leur statut résidentiel dans le cadre de la négociation salariale, ce qui donne à penser que leur stabilité leur permet d'accumuler une expérience spécifique valorisée en termes salariaux et/ ou de bénéficier d'effets favorables de leurs réseaux locaux. Au-delà, il faut aussi envisager que notre stratégie économétrique ne permet que de contrôler partiellement l'endogénéité du statut résidentiel : elle repose en particulier sur l'hypothèse d'absence de corrélation entre les caractéristiques inobservables qui pourraient simultanément influencer les décisions sur l'un et l'autre marché (5). Dans le cas où cette hypothèse serait réfutée, les résultats obtenus pourraient refléter les conditions relativement strictes en France de l'accès au crédit immobilier en fonction de la situation sur le marché $\mathrm{du}$ travail. Les recherches futures sur la question pourraient permettre d'éclaircir ce point en tenant compte de l'hétérogénéité inobservée et en intégrant explicitement la dynamique des salaires dans la modélisation.

En outre, l'extension de cette étude à d'autres pays pourrait être particulièrement enrichissante. Par exemple, une comparaison sur ce sujet entre la France et les États-Unis serait intéressante en ce sens qu'elle serait tout à fait emblématique de deux fonctionnements des marchés du travail et du logement opposant une régulation plutôt concurrentielle

(5) Selon les équations de l'annexe, on suppose que les termes d'erreur $u$ et $\varepsilon_{2}$ sont non corrélés. pour les États-Unis à une logique plus réglementée pour la France. Au-delà des effets spécifiques attendus dans chaque pays des contraintes rencontrées sur le marché du crédit et de la dynamique des prix et des loyers, la structure du marché du travail et son environnement macro-économique permettent d'envisager des liens différenciés entre statut résidentiel et salaires. D'ailleurs, BRUNET et al. (2011) ont mis en évidence que les propriétaires américains ne subissaient pas un risque de chômage significativement plus élevé que les locataires. Il est donc fort probable qu'aux États-Unis, aucune des parties en jeu dans la négociation salariale ne puisse tirer avantage d'une plus faible mobilité associée à la propriété immobilière et qu'au final, les statuts résidentiels ne jouent pas un rôle majeur dans les mécanismes d'ajustement de l'emploi sur le marché du travail américain. Des estimations semblables à celles de cette étude, que nous avons effectuées à partir des données américaines du Panel Study of Income Dynamics (PSID) 1994-1999, plaident dans le sens de cette absence de contagion du marché du logement sur le marché du travail. En cohérence avec les fonctionnements très flexibles de ces marchés et en particulier la faiblesse des coûts de transactions sur le marché immobilier Outre-Atlantique, la variable reflétant le statut résidentiel est en effet non-significative dans l'équation de salaire estimée sur le PSID (6). Néanmoins, ces résultats ont été obtenus en utilisant comme variable instrumentale pour le statut résidentiel individuel la proportion de propriétaires dans l'État, ce qui est discutable. C'est pourquoi, nos conclusions préliminaires pour les États-Unis mériteraient d'être confirmées sur d'autres données permettant d'avoir des instruments et donc des interprétations plus robustes.
(6) Les régressions effectuées à partir du PSID sont disponibles sur simple demande auprès des auteurs. 


\section{Bibliographie}

Battu H., Ma A., Phimister E. (2008), "Housing Tenure, Job Mobility and Unemployment in the UK", The Economic Journal, Vol. 118, № 257, pp. 311-328.

Belot M., EderveEn S. (2005), "Indicators of cultural and institutional barriers in OECD countries", $C P B$ Research Memorandum, The Hague.

Belot M., van OuRs J. (2001), "Unemployment and labor market institutions : An empirical analysis", Journal of the Japanese and International Economies, Vol. 15, № 4, Décember, pp. 403-418.

Blau F., Ferber M. (1987), "Discrimination : Empirical Evidence from United States", American Economic Review, Vol. 77, № 2, pp. 316-320.

Bosvieux J. (2005), « Accession à la propriété : des acquéreurs plus nombreux moins prudents », Économie et Statistique, vol. 381-382, pp. 41-62.

Breuil-Grenzier P., Valdelièvre H. (2001), « Le panel européen : l'intérêt d'un panel d'individus », Économie et Statistique, vol. 349-350, no 9/10, pp. 17-40.

Brunet C., Clark A., Lesueur J.-Y. (2007), « Statut résidentiel et durée de chômage en France et au Royaume-Uni », Revue française d'économie, vol. 22, no 2, pp. 165-190.

Brunet C., Havet N., Lesueur J.-Y. (2011), « La propriété immobilière est-elle un obstacle pour sortir $\mathrm{du}$ chômage ? », Économie et Prévision, à paraître.

Burgess S. (1992), "A search model with job changing costs : 'euroclerosis' and unemployment", Oxford Economic Papers, Vol. 44, № 1, pp. 75-88.

Catte P., Girouard N., Price R., André C. (2004), «Contribution des marchés du logement à la résilience économique », Revue économique de l'OCDE, vol. 38, $\mathrm{n}^{\mathrm{o}} 1$, pp. 139-173.

Coulson E., Fisher L. (2002), "Tenure Choice and Labour Market Outcomes", Housing Studies, Vol. 17, No 1, pp. 35-49.

Coulson E., Fisher L. (2009), "Housing Tenure and Labor Market Impacts : The Search Goes On”, Journal of Urban Economics, Vol. 65, № 3, pp. 252-264.

DAubresse M. (2003), « La reprise de l'accession à la propriété », INSEE Première, vol. 3, no 913, 4 pages.

de GraAfF T., van Leuvensteisn M. (2007), "The impact of housing market institutions on labour mobility : A European cross country comparison », CPB Discussion Paper 80.

De Graaff T., van Leuvensteijn, M., van EwiJK C. (2009), Homeownership, Social Renting and Labor Mobility accross Europe, in van Ewijk C., van Leuvensteijn M., "Homeownership \& the Labour Market in Europe", Oxford University Press, Chapter III, pp. 53-70.

Dubujet F., Blanc D. (2000), «Accession à la propriété immobilière : le régime de croisière ? », INSEE Première, vol. 3, no 718, 4 pages.

Gobillon L., Le Blanc D. (2005), « Quelques effets économiques du prêt à taux zéro ", Économie et Statistique, vol. 381-382, octobre, pp. 63-89.
Hausman J. (1978), "Specification Tests in Econometrics", Econometrica, Vol. 46, No 6, pp. 1251-1271.

Havet N. (2004), « Écarts salariaux et disparités professionnelles entre sexes : développements théoriques et validité empirique », L'Actualité économique, Revue d'analyse économique, vol. 80, pp. 3-31.

Havet N., Penot A. (2010), "Does Homeownership Harm Labour Market Performances ? A Survey", Working Paper du Gate, Vol. 1012, May, 29 p.

Holly A. (1982), “A Remark on Hausman's Specification Test”, Econometrica, Vol. 50, pp. 749-759.

Jovanovic B. (1979), "Job Matching and the Theory of Turnover", Journal of Political Economy, Vol. 87, No 5, pp. $972-980$.

LAFERRÈRE A. (2005), «Quitter le nid : entre forces centripètes et centrifuges », Économie et Statistique, vol. 381-382, octobre, pp. 147-175.

Manning A. (2003), Monopsony in Motion, Princeton, New Jersey, Princeton University Press.

Meurs D., Ponthieux S. (2000), «Une mesure de la discrimination dans l'écart de salaire entre hommes et femmes », Économie et Statistique, vol. 337-338, no 7/8, pp. $135-158$.

Mincer J. (1974), Schooling, Experience and Earnings, Columbia University Press for NBER, New York.

Munch J.R., Rosholm M., Svarer M. (2006), “Are Home Owners Really More Employed ?", The Economic Journal, Vol. 116, № 514, pp. 991-1013.

Munch J.R., Rosholm M., Svarer M. (2008), "Homeownership, Job duration and Wages", Journal of Urban Economics, Vol. 63, № 1, pp. 130-145.

Nickell S.,NunZiataL.,Ochel W.(2005), “Unemployment in the OECD since the 1960s. What do we know ?", The Economic Journal, Vol. 11, pp. 1-27.

Oettinger G. (1996) "Statistical Discrimination and the Early Career Evolution of the Black-White Wage Gap", Journal of Labor Economics, Vol. 14, № 1, pp. 52-78.

Oswald A. (1996), "A conjecture on the explanation for high unemployment in the industrialised nations : part 1", University of Warwick Economic Research Papers, 475.

Oswald A. (1998), "The missing piece of unemployment puzzle”, CEPR Workshop on Unemployment Dynamics.

VAN DEN BERG G. (1990), "Non Stationarity in job search theory", Review of Economic Studies, Vol. 57, No 2, pp. 2556277.

VAN VuUREN A. (2009), The impact of Homeownership on Unemployment in the Netherlands, in van Ewijk C., van Leuvensteijn M. "Homeownership \& the Labour Market in Europe", Oxford University Press, Chapter V, pp. 113-135.

WoOldRIDGe J.M. (2002), Econometric Analysis of Cross Section and Panel Data, The MIT Press. 


\section{Annexe \\ Estimations économétriques de l'influence du statut résidentiel sur le niveau des salaires}

\section{Estimations par moindres carrés ordinaires et par variables instrumentales :}

Le cadre généralement retenu pour évaluer l'impact de la propriété immobilière sur les rémunérations est une équation de salaire mincérienne incluant une dichotomique qui vaut 1 si l'individu est propriétaire de son logement et 0 sinon. Or, il est très probable que la variable de propriété immobilière soit endogène du fait de sa relation bidirectionnelle avec les salaires. Si tel est le cas, cette variable devra être « instrumentée » afin d'éviter que l'évaluation soit biaisée.

C'est pourquoi, nous avons estimé deux équations de salaire. La première introduit directement la variable dichotomique de propriété immobilière, considérée comme exogène, et est estimée par moindres carrés ordinaires :

$$
\ln \left(w_{i}\right)=X_{i} \beta_{1}+z_{i} \gamma_{1}+\varepsilon_{1 i}(\mathrm{MCO}),(1)
$$

où $w_{i}$ est le salaire horaire, $X_{i}$ représentent les caractéristiques socio-démographiques de l'individu $i$ et les caractéristiques de son emploi, $z_{i}$ vaut 1 si l'individu $i$ est propriétaire et 0 sinon et $\varepsilon$ est le terme d'erreur.

La deuxième équation introduit à la place de la variable $z_{i}$ la probabilité individuelle d'être propriétaire, $\hat{P}\left(z_{i}=1\right)$, estimée à l'aide d'un probit dans une étape antérieure, afin de pallier à son éventuel caractère endogène (méthodes des variables instrumentales) :

$\ln \left(w_{i}\right)=X_{i} \beta_{2}+\hat{P}\left(z_{i}=1\right) \gamma_{2}+\varepsilon_{2 i}$ (Variables instrumentales) (2).

L'équation (2) est estimée par MCO avec l'estimateur de variance de type Huber-White qui permet de corriger les problèmes d'hétéroscédasticité de la méthode des variables instrumentales et de tenir compte des éventuelles corrélations entre les observations associées à un même individu.

Plus précisément, on a estimé précédemment par maximum de vraisemblance le modèle probit :

$z_{i}=\left\{\begin{array}{l}1 \text { si } z_{i}^{*}=W_{i} \alpha+u_{i} \geq 0 \\ 0 \text { sinon }\end{array}\right.$

avec $W_{i}$ les déterminants de la propriété immobilière, qui doivent contenir au moins une variable significative non incluse dans $X_{i}$ (ici la variable d'héritage et les variables reflétant les montants moyens des taxes locales) et $u_{i}$ un terme d'erreur suivant une loi normale centrée réduite.
La probabilité $\hat{P}\left(z_{i}=1\right)$ a alors pour expression : $\hat{P}\left(z_{i}=1\right)=\Phi\left(W_{i} \hat{\alpha}\right)$ où $\Phi$ est la fonction de répartition de la loi normale centrée réduite et les coefficients estimés du modèle probit.

Test de l'endogénéité de la variable de propriété immobilière dans l'équation de salaire

Pour déterminer laquelle de ces deux estimations (équations 1 et 2 ) est la plus pertinente, il faut tester si la variable de propriété immobilière est exogène ou endogène dans l'équation de salaire. Ce test est effectué par le biais de la régression augmentée basée sur le test de spécification de Hausman. Il s'agit d'effectuer une régression artificielle où l'on introduit dans l'équation de salaire la variable dichotomique de propriété immobilière $z_{i}$ et sa variable prédite par la régression probit $P\left(z_{i}=1\right)$, comme suit :

$\ln \left(w_{i}\right)=X_{i} \beta_{3}+z_{i} \gamma_{3}+\hat{P}\left(z_{i}=1\right) \delta+\varepsilon_{3 i}$ (régression augmentée).

L'hypothèse d'endogénéité est rejetée si la variable $P\left(z_{i}=1\right)$ est significative ou en d'autres termes si le coefficient $\delta$ est statistiquement différent de zéro. Dans ce cas, il faudra privilégier la méthode par variables instrumentales qui tient compte de l'endogénéité de la variable de statut résidentiel. Dans le cas contraire, l'estimation par MCO sera la plus pertinente car elle donne des résultats non biaisés et plus précis que la méthode des variables instrumentales.

À partir de nos données du PEM, nous obtenons les résultats reportés dans le tableau 3. Il montre sans ambiguïté que l'hypothèse nulle d'exogénéité est rejetée puisque la variable prédite de propriété immobilière est significative au seuil de $5 \%$. Ainsi, il faut privilégier l'estimation de l'équation (2) par la méthode des variables instrumentales, l'estimation de l'équation (1) étant biaisée.

Tableau 3 : Test d'endogénéité

\begin{tabular}{|l|c|c|c|}
\hline \multicolumn{1}{|c|}{ Variables } & $\begin{array}{c}\text { Coef. } \\
\text { estimé }\end{array}$ & $\begin{array}{c}\text { Ecart- } \\
\text { type }\end{array}$ & P-value \\
\hline Propriétaire $: z_{i}$ & 0,053 & 0,009 & 0,000 \\
\hline Proba. prédite $: \hat{P}\left(z_{i}=1\right)$ & 0,101 & 0,047 & 0,033 \\
\hline
\end{tabular}

